\title{
Target-Oriented Coverage Maximization in Visual Sensor Networks
}

\author{
Vikram P. Munishwar \\ Computer Science \\ State University of New York at Binghamton \\ vmunish1@cs.binghamton.edu
}

\author{
Nael B. Abu-Ghazaleh \\ Computer Science \\ State University of New York at Binghamton \\ nael@cs.binghamton.edu
}

\begin{abstract}
Visual sensor networks (VSNs) are networks made up of a possibly large number of cameras that together monitor an area or targets of interest. In VSNs, camera coverage control is necessary to allow automatic tracking of targets without human intervention, allowing these systems to scale. In this paper, we consider the problem of automatic control of cameras to maximize the number of targets covered. This is an NP-hard problem, and efficient centralized and semi-centralized heuristics exist that provide near-optimal performance. However, such schemes are hard to scale with the size of the network, and thus demand for efficient distributed solutions. The existing distributed approach results in significantly less coverage, compared to the optimal. In this paper, we extend the existing approach by considering inter-dependencies among cameras and target distributions, and show that the proposed techniques significantly improve the performance of the distributed solution.
\end{abstract}

\section{Categories and Subject Descriptors}

C.2.1 [Computer-Communication Networks]: Network Architecture and Design-Wireless communication, Distributed networks

\section{General Terms}

Algorithms, Design, Performance

\section{Keywords}

Camera sensors, Coverage, Distributed protocol

\section{INTRODUCTION}

Recent technological developments in processing, imaging and communication have led to the development of smart

\footnotetext{
${ }^{*}$ This research work is funded by Qatar National Research Fund (QNRF) under the National Priorities Research Program (NPRP) Grant No.:08-562-1-095
}

Permission to make digital or hard copies of all or part of this work for personal or classroom use is granted without fee provided that copies are not made or distributed for profit or commercial advantage and that copies bear this notice and the full citation on the first page. To copy otherwise, to republish, to post on servers or to redistribute to lists, requires prior specific permission and/or a fee.

MobiWac'11, October 31-November 4, 2011, Miami, Florida, USA.

Copyright 2011 ACM 978-1-4503-0901-1/11/10 ...\$10.00. camera nodes that can operate autonomously and collaboratively to meet an application's requirements. Networks of such cameras, called Visual Sensor Networks (VSNs) [10], have a range of applications in areas such as monitoring and surveillance, traffic management and health-care [2]. The self-configuring nature of cameras coupled with their processing ability and wireless connectivity enable them to become a part of the existing fixed infrastructure of surveillance cameras, or to be deployed for ad hoc surveillance in situations where infrastructure is unavailable or expensive, or quick deployment is desired.

The cameras must be controlled (pan, and if available, tilt and zoom) to provide the best possible coverage of events happening within the area of interest. As the scale of cameras grows from tens to hundreds of cameras, it is impractical to rely on humans to control their setting to achieve the best combined coverage. Thus, supporting autonomous configuration of cameras to maximize their collective coverage is a critical problem in smart camera networks. Specifically, the goal here is to maximize the number of targets covered by all cameras, where cameras can cover only a limited spherical sector (in 3-D) of the sphere of their potential coverage region at a given time. We term this problem as a target-oriented coverage maximization problem and it has been defined formally in the literature $[1,7]$. Other variations, but with different objectives, of the coverage problem exist as well. For instance, the Art Gallery Problem [12] and its variations focus on the optimal placement of cameras to maximize the coverage of (or fully cover) the area of interest. In settings where cameras are pre-deployed, cameras' sensing directions (pans) can be adjusted to maximize the total area under coverage $[6,11]$. In addition, for surveillance class applications, camera FoVs are changed according to a schedule that maximizes coverage of targets over time $[5,8]$.

Target-oriented coverage maximization problem is NPhard, and efficient centralized and semi-centralized heuristics exist that can provide near-optimal performance $[1,7]$. While, centralized heuristics can provide close-to-optimal results, they incur substantial networking overhead. Specifically, in centralized mechanism, all cameras send their coverage information to a central node (a.k.a. base-station), which computes the best configuration (pan) for each camera, which can collectively maximize the number of targets covered. The communication latency in centralized approaches increases drastically as the density of cameras increases [7], rendering them unsuitable for large-scale networks. On the other hand, distributed approaches typically 
involve message communication only with neighbors, and thus are easily scalable.

However, distributed protocols for target coverage maximization have received relatively less attention. The existing protocol, Distributed Greedy Approach (DGA) [1] is a greedy heuristic that tries to maximize the coverage by coordinating with neighboring cameras. However, it achieves significantly less coverage compared to the optimal and centralized heuristics [7]. We describe the problem and the DGA approach in detail in Section 2.

Thus, in this paper, we focus on increasing the overall coverage gain achieved by a distributed approach. More specifically, we extend the DGA to take into account interdependencies among cameras and target distributions. DGA assigns unique random priorities to cameras, which help the distributed approach to converge. However, the randomness in priority assignments can lead to significant loss of coverage, as it completely ignores the camera placement scenario and their inter-dependencies, as well as the distribution of targets. We use the degree-of-overlap among cameras and distribution of targets of interest, as metrics to develop priority assignment schemes for cameras. Section 4 presents performance evaluation, which shows that the proposed priority assignment schemes result in around $5 \%$ to $10 \%$ increase in the overall coverage gain. Section 5 presents some concluding remarks and directions for future work.

\section{COVERAGE MAXIMIZATION PROBLEM}

In this section, we first present our assumptions and then formally define the target-oriented coverage maximization problem. Finally, we describe the existing distributed approach to address the problem.

We make the following assumptions in this paper: (1) Target localization: Like prior work in the directional sensor target maximization, we assume that the location of the targets is determined using complementary mechanisms such as, stereoscopic localization [4], and LAser Detection And Ranging device (LADAR)-based localization [13]. (2) Cameras: For this work, we consider a camera node as a Panonly camera. (3) Targets: We assume that targets are covered if a selected point on them is covered; a target is either completely covered or not covered. This point can be the centroid of the target, for example.

\subsection{Problem Definition}

The target-oriented coverage maximization problem can be formally defined as follows. Consider a set of static cameras, $N$, trying to monitor a set of static targets of interest, $M$, present in an area free of obstacles. Each camera can be configured to take one pan from the possible discrete set of pans, $P$. Thus, the problem can be defined as: how to assign a pan $p \in P$ to each camera $c \in N$ such that the collective number of targets $t \in M$ covered is maximized?

Target-oriented coverage maximization problem is shown to be NP-hard, and efficient centralized and semi-centralized heuristics exist that provide near-optimal coverage $[1,7]$. However, the existing distributed coverage maximization heuristic, which we explain next, results in a significant loss of coverage.

\subsection{Existing Solution}

In the existing solution, Distributed Greedy Approach (DGA) [1], all sensors assign themselves a unique priority value, and initialize their direction in a random manner. Each sensor detects the total number of targets it can cover in each direction, and selects the direction covering maximum targets. It then sends this direction information to its sensing neighbors (neighbors within $2 *$ sensing range distance). Overlaps in the target coverage are avoided by accepting the decision of higher priority sensor node. The priority assignment scheme of DGA ensures that the algorithm will terminate. Specifically, the algorithm converges in iterative fashion, stabilizing from the highest priority node in the network.

While unique priority assignment in DGA ensures its convergence, the randomness in priority assignment can lead to highly sub-optimal coverage. Thus, in this work, we propose better priority assignment schemes to improve overall coverage utility.

\section{EXTENSIONS TO DGA}

In this section, we describe extensions to the existing Distributed Greedy Approach by proposing overlap-based and target-distribution based camera priority assignment schemes.

\subsection{Area-based Priority Assignment}

Coverage maximization approaches are required only when there is inter-dependence among cameras. Essentially, when there is an overlap in cameras' coverage area, their FoVs must be configured such that they do not cover common targets. Thus, while a camera with no coverage overlaps with the other cameras can set its FoV in greedy manner, the camera's dependence on other cameras increases as it shares the coverage region with more cameras. We use this insight and allocate higher priority to a camera having lower degrees of coverage overlaps with the other cameras. Thus, for a camera $c \in N$, its priority, $c_{p r}$, can be set as:

$$
c_{p r}=|N|-\left|S N_{c}\right|
$$

where, $S N_{c}$ is a set of Sensing Neighbors of camera $c$. Recall that the sensing neighbors of a camera are the cameras located within its $2^{*}$ sensing range distance.

\subsection{Target-based Priority Assignment}

While area-based overlaps may expose camera dependencies at a broader level, it may also result in false-positives, since there may not be any target in the overlapped region. Thus, in this approach, we attempt to derive camera priority based on the targets it can cover.

Essentially, a camera can choose a pan from the set of discrete pans, $P$. However, not all of the possible pans may be useful-some of them may not cover any target. Thus, it is sufficient for a camera to choose a pan only from the useful set of pans. In general, target distribution can affect the total number of useful pans a camera can have. If the targets are clustered into one pan, the camera will have only one useful pan. Thus, the camera can greedily select the pan, irrespective of the decisions of the other cameras. However, as the targets are distributed more uniformly, the number of useful pans increases, which complicates the pan selection process due to the increased dependence on the sensing neighbors. Thus, a camera's priority is higher if it has lower number of useful pans, and it can be computed as:

$$
c_{p r}=|P|-\left|F P_{c}\right|
$$




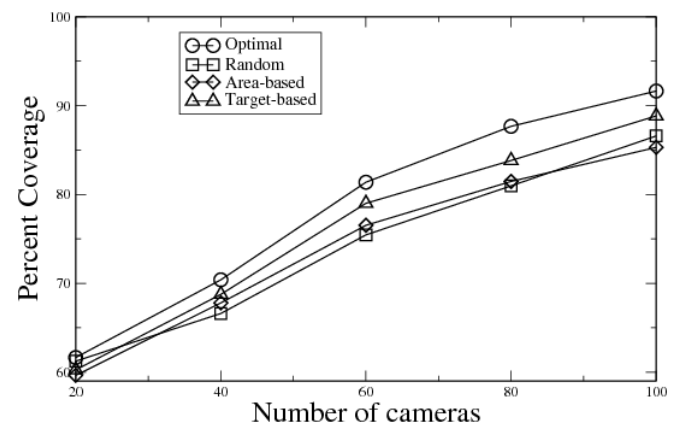

Figure 1: Impact of varying camera density on coverage gain.

where, $F P_{c}$ represents the set of feasible (or useful) pans for camera $c \in N$.

Tie-breaking procedure: The DGA with random priority scheme ensures that the cameras receive unique priorities. However, the Area- and Target-based priority assignment schemes may not adhere to the unique priority requirement. Thus, we slightly modify the DGA algorithm for a camera to accept packets from a neighbor even if they both have the same priority, and apply tie-breaking mechanisms to decide whether to accept or discard the packet. Specifically, if both the cameras have the same priority, the camera that covers higher number of targets than the other camera wins. If they both cover the same number of targets, then we break the tie by favoring a camera having a higher Id number.

\section{PERFORMANCE EVALUATION}

We compare DGA with different priority assignment schemes for varying camera densities and target distributions. We term the existing DGA protocol with random priority assignment as Random, and its extensions for area-based and target-based priority assignments as Area-based and Targetbased, respectively. We also show their performance with respect to the Optimal policy, which essentially runs the optimization problem to find camera-pan pairs that collectively maximize the total targets covered $[1,7]$.

To evaluate the above approaches, we have extended the QualNet simulator [9] to model cameras and their field-ofviews (FoVs). Each camera is set with a pre-defined maximum coverage range, $R_{\max }=100$ meters, a minimum coverage range, $R_{\text {min }}=0$ meters, and an FoV angle, $\theta=45^{\circ}$. The camera can choose from 8 discrete pan values as its orientation, however the proposed approaches can use higher number of pans, which could be overlapping.

\subsection{Impact of camera density}

We change camera density by keeping the terrain size fixed as $1000 \times 1000$ sq. meters, and changing the number of cameras deployed on the terrain. We deploy cameras using uniform random distribution. For this study, we deploy 100 targets on the terrain using random distribution. We evaluate the sensitivity of observed coverage gain and convergence delay to camera density. We represent the coverage gain as Percent Coverage, which is a ratio of the total covered to coverable (within coverage range) targets.

As it can be noted in Figure 1, the proposed priority assignment, especially the Target-based scheme, leads to better

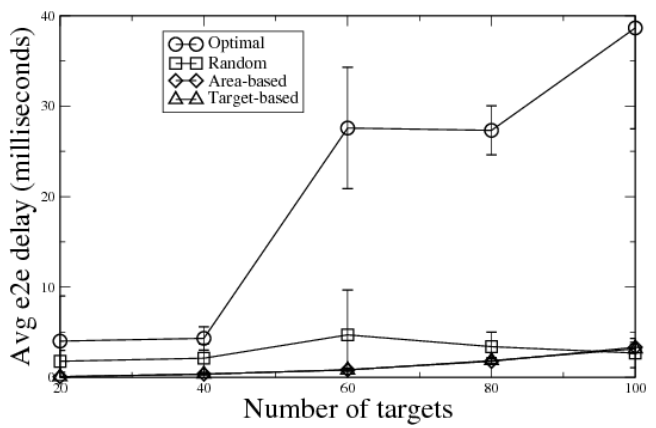

Figure 2: Impact of varying camera density on convergence time.

coverage gain (and follows Optimal more closely) compared to random priority assignment, in most of the cases. There can be a few scenarios where the Random appears to perform slightly better than the proposed approaches, but we conjecture that this is an artifact of the randomness in priorities and target deployments. Figure 2 compares the convergence time for the protocols, which shows that the proposed priority assignment schemes lead to lower convergence time compared to that of the Random. It is also evident that the overhead for the Optimal approach increases drastically with the camera density, due to its centralized nature [7].

\subsection{Impact of target distribution}

To study the impact of different potential target distributions possible in surveillance applications, we consider random, uniform, and clustered targets distributions. Figure 3, Figure 4, and Figure 5 represent the uniform, moderatelyand strongly-clustered distributions of targets used in this experiment, respectively. These distributions are generated using the existing approach to generate inhomogeneous node distributions [3]. The number of cameras set for this experiment is 100 .

Figure 6, Figure 7, and Figure 8 plot the overall coverage gain for DGA and its extensions for uniform (non-clustered), moderately-clustered and strongly-clustered target distributions, respectively. As it can be noted from the graphs, the proposed priority assignment schemes clearly outperform the Random approach. In fact, the Target-based scheme provides closer-to-optimal results as the clustering of targets becomes stronger. This behavior is due to the fact that the strong clustering of targets reduces the number of feasible pans for all the cameras, in general. Thus, some cameras get only one feasible pan, and hence the highest priority. This causes the lower-priority neighbors of these cameras to ignore the targets already covered by higher priority cameras and select the pan that covers the most of the remaining targets. It is also possible for camera neighbors to receive high but equal priorities. This leads them to use the tie-breaking mechanism, which favors camera-pan pairs that cover higher number of targets. Thus, both the cases directly lead to increasing the overall coverage gain.

\section{CONCLUDING REMARKS}

In this paper, we consider the problem of automatic control of the cameras to maximize coverage of a set of targets. This problem is known to be NP-hard, and efficient central- 


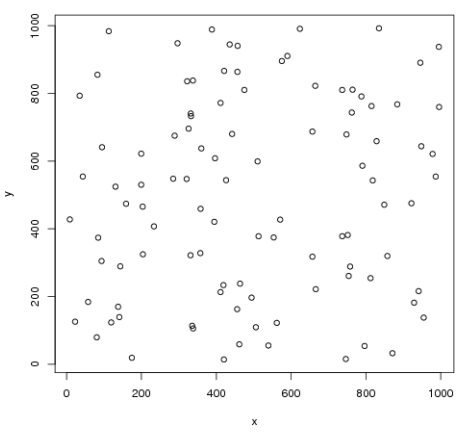

Figure 3: No Clustering

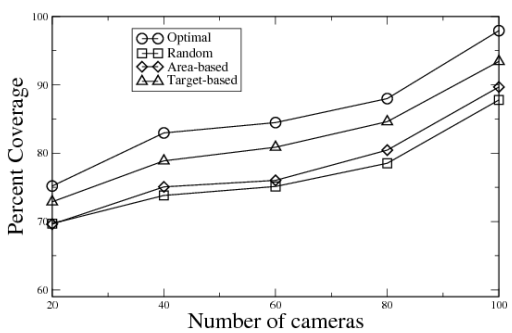

Figure 6: Coverage Gain for No Figure 7: Coverage Gain Clustering Case.

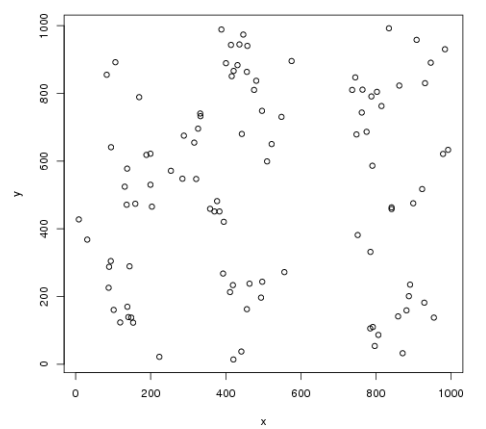

Figure 4: Medium Clustering

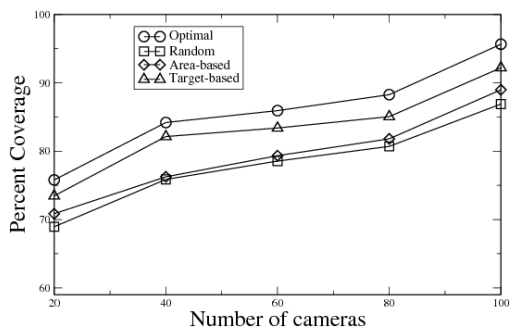

Medium Clustering Case.

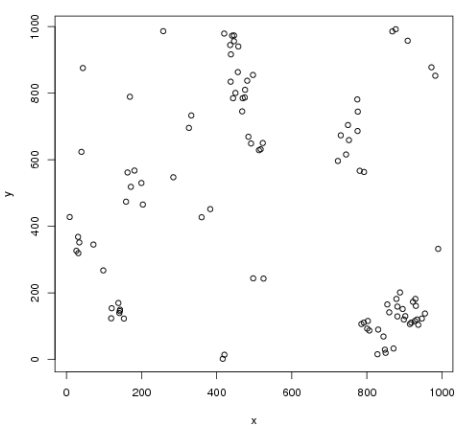

Figure 5: Strong Clustering

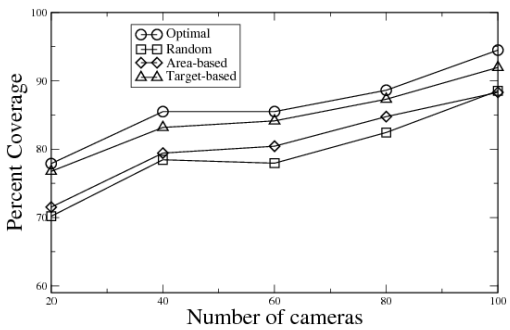

for Figure 8: Coverage Gain for Strong Clustering Case. ized and semi-centralized heuristics exist that perform closeto-optimal. However, such approaches are hard to scale, and thus distributed protocols are sought-after. The existing distributed approach, Distributed Greedy Approach (DGA), results in significant loss of coverage. We extended DGA by developing novel schemes for camera priority assignment. The proposed Target-based scheme results in up to $10 \%$ of increase in the overall coverage gain.

As a continuation, we plan to investigate the use of the notion of force the targets exert on a pan of a camera [7] (which gives near-optimal performance for centralized approaches) in a distributed setting. We also plan to study the impact of target mobility on the distributed approaches and propose mechanisms to handle it efficiently.

\section{REFERENCES}

[1] J. Ai and A. Abouzeid. Coverage by directional sensors in randomly deployed wireless sensor networks. Journal of Combinatorial Optimization, 2006.

[2] I. Akyildiz, T. Melodia, and K. Chowdhury. A survey on wireless multimedia sensor networks. Computer Networks, 51(4):921-960, 2007.

[3] C. Bettstetter, M. Gyarmati, and U. Schilcher. An inhomogeneous spatial node distribution and its stochastic properties. In Proceedings of the 10th ACM Symposium on Modeling, analysis, and simulation of wireless and mobile systems, page 404. ACM, 2007.

[4] M. Garcia and A. Solanas. 3D simultaneous localization and modeling from stereo vision. In Robotics and Automation, 2004. Proceedings. ICRA'04. 2004 IEEE International Conference on, volume 1, pages 847-853. IEEE, 2004.

[5] N. Krahnstoever, T. Yu, S. Lim, K. Patwardhan, and P. Tu. Collaborative Real-Time Control of Active
Cameras in Large Scale Surveillance Systems. In Workshop on Multi-camera and Multi-modal Sensor Fusion Algorithms and Applications, 2008.

[6] H. Ma, X. Zhang, and A. Ming. A coverage-enhancing method for $3 \mathrm{~d}$ directional sensor networks. In Proc. INFOCOM Mini-conference, pages 2791-2795, 2009.

[7] V. Munishwar and N. Abu-Ghazaleh. Scalable Target Coverage in Smart Camera Networks. In ACM/IEEE International Conference on Distributed Smart Cameras, 2010.

[8] F. Z. Qureshi and D. Terzopoulos. Surveillance camera scheduling: a virtual vision approach. In VSSN '05: Proceedings of the third ACM international workshop on Video surveillance $\&$ sensor networks, pages 131-140, New York, NY, USA, 2005. ACM.

[9] Q. Simulator. Scalable Network Technologies. Inc.[Online]. Available: www. qualnet. com.

[10] S. Soro and W. Heinzelman. A survey of visual sensor networks. Advances in Multimedia, (640386):1-21, 2009.

[11] D. Tao, H. Ma, and L. Liu. Coverage-enhancing algorithm for directional sensor networks. Lecture Notes in Computer Science, 4325/2006:256-267, 2006.

[12] J. Urrutia. Art gallery and illumination problems. Handbook of Computational Geometry, pages 973-1027, 2000.

[13] C. Wang, C. Thorpe, and A. Suppe. Ladar-based detection and tracking of moving objects from a ground vehicle at high speeds. In Proceedings of Intelligent Vehicles Symposium. IEEE, 2003. 